\title{
B cell non-Hodgkin's lymphoma in a girl with the DiGeorge anomaly
}

\author{
Jose T Ramos, Eduardo López-Laso, Jesus Ruiz-Contreras, Elisa Giancaspro, \\ Santiago Madero
}

\begin{abstract}
The DiGeorge anomaly (DGA) is occasionally associated with cellular immunodeficiency. We report a female infant diagnosed with complete DGA, who developed fatal, high grade, non-Hodgkin's lymphoma that expressed Epstein-Barr virus (EBV). Non-Hodgkin's lymphoma should be considered in children with DGA.

(Arch Dis Child 1999;81:444-445)
\end{abstract}

Keywords: DiGeorge anomaly; Epstein-Barr virus; B cell lymphoma; CATCH 22

The DiGeorge anomaly (DGA) is an uncommon developmental defect resulting from an abnormal migration of neural crest elements involving predominantly the third and fourth pharyngeal pouches, leading to a variety of congenital heart defects, hypoplasia of the thymus and parathyroid glands, and characteristic dysmorphic features. ${ }^{1}$ Molecular analysis has demonstrated deletion in chromosome $22 \mathrm{q} 11 .{ }^{2}$ Approximately $19-25 \%$ of patients diagnosed with the DGA have a $\mathrm{T}$ cell immunodeficiency. ${ }^{3}$

Children with $\mathrm{T}$ cell immunodeficiencies are

Division of

Immunodeficiencies,

Department of

Pediatrics, Hospital 12

de Octubre, Cra.

Andalucia Km 5,400

28041 Madrid, Spain

J T Ramos

E López-Laso

J Ruiz-Contreras

E Giancaspro

Division of Pathology, Hospital 12 de Octubre S Madero

Correspondence to: Dr Ramos.

Email: jramos@h12o.es

Accepted 4 March 1999 prone to the development of lymphoid neoplasia. ${ }^{4}$ Epstein-Barr virus (EBV) has been associated with B cell non-Hodgkin's lymphomas in immunocompromised patients. Despite the severe immunodeficiency in some children with DGA, we could not find any previous cases of B cell non-Hodgkin's lymphoma in patients with DGA in the English literature.

\section{Case report}

A 10 month old female patient was admitted with a seven day history of severe vesicular rash and fever. She was the second child of a healthy 32 year old woman. She had normal development and her weight and height were at the 25th and 50th centiles, respectively. Initial

Table 1 Evolution of lymphocyte subsets

\begin{tabular}{llccc}
\hline Lymphocytes & Normal & 11 months & 14 months & 20 months \\
\hline Total $\left(\times 10^{6} / 1\right)$ & $2.6-11.8$ & 1.9 & 3.3 & 1.2 \\
CD2 (\%) & $86-99$ & 90 & 68 & 83 \\
CD3 (\%) & $56-85$ & 9 & 9 & 11 \\
CD4 (\%) & $29-54$ & 14 & 5 & 3 \\
CD8 (\%) & $11-40$ & 9 & 14 & 17 \\
CD28 (\%) & 85 & 7 & NA & NA \\
CD25 (\%) & $1-12$ & 17 & 32 & 0 \\
CD19 (\%) & $9-34$ & 54 & 12 & 46 \\
CD21 (\%) & $4-19$ & 20 & 7 & 35 \\
CD57 (\%) & $2-20$ & 13 & 43 & 33 \\
CD16 (\%) & $1-17$ & 63 & & \\
\hline
\end{tabular}

^Normal values according to our hospital immunology unit.

NA, Not available examination revealed a febrile, toxic appearing, pale infant with a generalised vesicular rash consistent with chickenpox. She had no dysmorphic features or cardiac anomalies. The absolute lymphocyte count was $1.9 \times 10^{6} / 1$, calcium concentration was $1.5 \mathrm{mmol} / 1$, and ionised calcium $0.97 \mathrm{mmol} / 1$. Alanine (ALT) and aspartate (AST) aminotransferases were moderately increased (ALT, 140; AST, $164 \mathrm{IU} / 1)$. No thymic shadow could be seen on chest roentgenogram; the patient was HIV antibody negative.

She was treated with antibiotics and aciclovir and made an uneventful recovery. Subsequently, parathormone level was $6.7 \mathrm{pg} / \mathrm{ml}$ (normal value in our laboratory: $10-67 \mathrm{pg} / \mathrm{ml}$ ). The assessment of immunological status showed CD4 and CD8 lymphocyte counts of 0.27 and $0.17 \times 10^{6} / 1$, respectively. B lymphocyte count and immunoglobulin levels were within normal limits. Skin hypersensitivity tests were anergic. Phytohaemagglutinin response was decreased ( $7 \%$ relative to control). Subsequent immunological evaluation is shown in table $1 .^{5}$

The patient was discharged with a diagnosis of complete DGA on the basis of $\mathrm{T}$ cell deficiency and hypoparathyroidism. She was treated as an outpatient with oral calcium, vitamin D, and cotrimoxazole. Several episodes of thrush and suppurative otitis media occurred during follow up. She was immunised with pneumococcus polysaccharide vaccine and conjugated Haemophilus influenzae b vaccine. After 30 days there was no change in serum antibody titres to these antigens. No karyotyping or study for $22 \mathrm{q} 11$ deletion were performed.

At the age of 21 months an abdominal ultrasound as assessment of hypocalcaemia showed a hyperechogenic mass in the liver. A laparoscopy with biopsy of the mass showed extensive necrosis and a non-specific inflammatory infiltrate. At 23 months she developed a progressive right hemiparesis. Physical examination revealed an alert child, evidence of spastic right hemiparesis, and conjugated gaze deviation to the left. Cranial computed tomography and cranial magnetic resonance imaging (fig 1) disclosed a large left cerebral hemispheric mass, two smaller space occupying lesions in the right basal ganglia, and scattered subcortical calcification. A sterotactic brain biopsy was done revealing a lymphoplasmocytary infiltrate.

Immunoblastic B cell non-Hodgkin's lymphoma was diagnosed cytomorphologically and by immunophenotyping. Capsid and nuclear antigen EBV antibodies were negative 


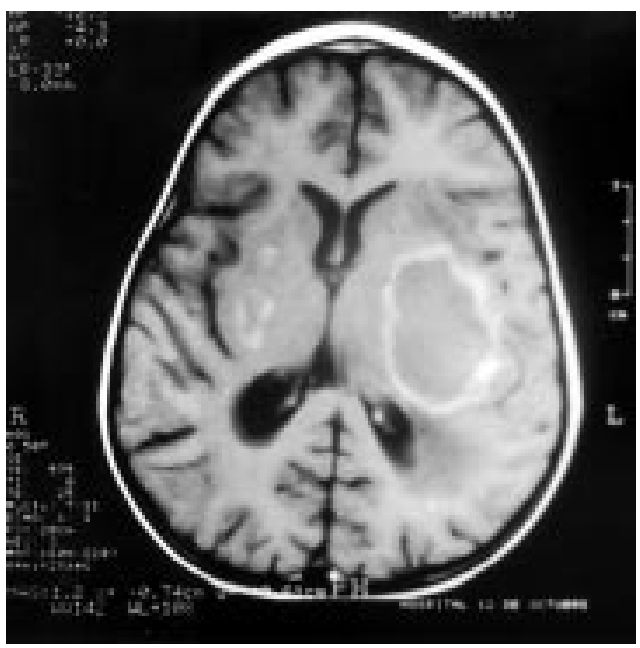

Figure 1 Transverse axial T1 weighted magnetic resonance imaging after infusion of paramagnetic contrast shows multiple ring enhancement lesions in cerebral hemispheres.

at the age of 19 and 23 months. In situ hybridisation showed a massive expression of EBV EBER (EBV encoded small RNA) transcripts in the lymphoma tissue. HLA haplotype of the patient's parents and brother were histoincompatible. The child's parents refused treatment of the malignancy. The patient became increasingly unresponsive and died one month later. The postmortem diagnosis was: absence of thymus and parathyroid glands; absence of cardiovascular defects; immunoblastic nonHodgkin's B cell lymphoma involving mediastinal lymph nodes, brain, liver, and both kidneys.

\section{Discussion}

DGA is an uncommon congenital malformation arising early in embryonic life. Although familial cases have been described most are sporadic. Recent availability of molecular techniques have shown a microdeletion of the $22 \mathrm{q} 11$ region in most cases of DGA, resulting in a wide range of phenotypes. ${ }^{2}$

Although arbitrary, patients with DGA may be classified into two groups on the basis of cellular immunodeficiency. The partial form of DGA, the most common one, is characterised by a preserved number and function of $\mathrm{T}$ cells, whereas the complete form is defined by severe cellular immunodeficiency. DGA was suspected in our patient because of unusually severe chickenpox and hypocalcaemia. Immunological studies showed a progressive decline in $\mathrm{T}$ cell number and function. The specific production of antibodies was also impaired as suggested by negative EBV serology despite a definite infection. Similarly to solid organ transplant recipients, in the setting of cellular immunodeficiency, a primary infection by EBV may lead to the rapid development of monoclonal lymphoproliferation.

Children with congenital immunodeficiencies have an increased risk for lymphoid neoplasia. Most reported cases of lymphoma in primary immunodeficiencies are high grade $B$ cell lymphomas with extranodal involvement. ${ }^{4}$ The risk for other malignancies in DGA patients is unknown. A single case of glioma has been reported by Asamoto and Furuta. ${ }^{6}$ To the best of our knowledge no other tumours have been reported in association with DGA. Nevertheless, the severe $\mathrm{T}$ cell immunodeficiency makes these patients prone to early development of lymphoproliferative syndromes should EBV infection occur. Treatment of the immunodeficiency associated with DGA is uncertain. Bone marrow transplantation from HLA matched siblings has successfully been performed, but there is limited experience. ${ }^{7}$ In cases of associated anomalies or a family history of the syndrome, detection of the characteristic microdeletion of the 22q11 region may be important for early diagnosis. If other cases of malignant complications are reported, this would strengthen the case for bone marrow transplantation in selected cases.

We thank Dr Carlos V Paya and Dr Pat Roche (Division of Infectious Diseases, Mayo Clinic, Rochester, Minnesota, USA) for kindly performing EBV in situ hybridisation. We also are in debted to Alfredo Corell (Division of Immunology, Hospital 12 de Octubre) for performing the immunological studies and for technical assistance.

1 Muller W, Peter HH, Wilken M, et al The DiGeorge syndrome I. Clinical evaluation and course of partial and
complete forms of the syndrome. Eur $\mathcal{f}$ Pediatr 1988;147:496-501.

2 Webber SA, Hatchwell E, Barber JC, et al. Importance of microdeletions of chromosomal region $22 \mathrm{q} 11$ as a cause of selected malformations of the ventricular outflow tracts and aortic arch: a three-year prospective study. F Pediatr

3 Bastian J, Law S, Vogler L, et al. Prediction of persistent mmunodeficiency in the DiGeorge anomaly. 7 Pediatr 1989;115:391-6.

4 Mueller B, Pizzo $\mathrm{Ph} \mathrm{A}$. Cancer in children with primary or secondary immunodeficiencies. F Pediatr 1995;126:1-10.

5 Regueiro JR, López-Botet M, Landazuri MO, et al. An in vivo functional immune system lacking polyclonal T-cell surface expression of the CD3/Ti (WT31) complex. Scand f Immunol 1987;26:299-302.

6 Asamoto H, Furuta M. DiGeorge syndrome associated with glioma and two kinds of infection [letter]. $N$ Engl f Med 1977;296:1235.

7 Borzy MS, Ridgway D, Noya FJ, Shearer WT. Successful bone marrow transplantation with split lymphoid chimerism in DiGeorge syndrome. F Clin Immunol 1989;9:38692 